\title{
Innovation off the beaten path
}

\author{
The Nature/Entrepreneur First Innovation Forum in Quantum Technologies was an experiment in \\ entrepreneurial thinking for young scientists.
}

Nature and its sister journals have a reputation for publishing cutting-edge fundamental results and we strongly believe that expanding knowledge is in itself a convincing reason to do science. Likewise, it is important to remember that by-products of fundamental research in the form of innovations can be enormously beneficial for society. There wouldn't be radios if Maxwell hadn't been interested in electromagnetic waves just for the sake of it. Without the fundamental interest of people such as John Stewart Bell and David Deutsch in quantum physics, quantum information applications would not be moving towards commercialization today.

As fields such as quantum information move towards more applied research, and focus on the commercialization of many decades of fundamental science, we want to assist beyond simply publishing on these developments. Inspiring and fostering entrepreneurial thinking among young scientists is a critical ingredient for the success of commercialization efforts, hence we conceived and organized the Nature/Entrepreneur First Innovation Forum in Quantum Technologies. In collaboration with Innovate UK, we selected five groups of young physicists in an open application process. Entrepreneur First, a London-based company that helps people with skills in technology to found start-ups, helped the young physicists to assess and polish their business ideas. And, on 6 May 2016, in a grand finale, these young minds got the opportunity to discuss their ideas with a prominent panel of experienced entrepreneurs and academics. Ying Lia Li, one of those selected, describes her experience on page 652 .

This project was an experiment for Nature and its sister journals and we cannot deny a certain nervousness about its outcome. But listening to the students' presentations, which ranged from unconditionally secure signatures to a silicon-based quantum computer (http://go.nature.com/28IVjGO), and witnessing the constructive and vibrant discussion about trajectories that quantum technology start-ups could take, about common pitfalls and overlooked opportunities, and about the grit and courage that it takes to found a start-up in a new and largely unexplored technology, we feel that we have indeed contributed to nurturing entrepreneurial thinking.

And in addition to inspiring young scientists to become business founders, the Nature/Entrepreneur First Innovation Forum has injected enthusiasm within our editorial teams, kindling interest in entrepreneurship and in projects that do not follow the beaten path.

\section{What is Nano?}

\section{A comprehensive nanotechnology-specific database has been launched as part of the Nature Research portfolio.}

On 15 June 2015, Springer Nature launched Nano, the first non-journal-type product to be marketed by the company within the Nature Research portfolio. Nano is a database, but it is also a discovery tool. It is designed to provide researchers in academia and industry a simple way to retrieve information on nanomaterials and nanodevices. Over 200,000 profiles have been created, and each is based on information extracted mainly from research articles published in 30 journals. By searching the database, users are presented with at-a-glance information on different types of materials or devices related to the keyword used, including composition and properties, and including the source articles and patents from which the information has been extracted.

Realizing a comprehensive catalogue of nanomaterials and nanodevices is important, particularly now, as after a few decades of intense research to understand the fundamental properties of nanostructures, efforts have now shifted to incorporate such structures in commercial devices. But creating a comprehensive catalogue of nano-objects is no easy task. The main challenge is, and possibly will always be, deciding what goes in and what stays out. What size can be used as a threshold under which a material becomes a nanomaterial? The only useful answer is that a nanomaterial has qualitatively different physical and chemical properties from its bulk counterpart, and the size at which this happens varies with each material. To complicate matters, nanomaterials are studied by physicists, engineers, chemists and biologists, and information is scattered in a wide variety of publications.
A product such as Nano can help. By collecting information from research articles and patents, it follows the definition of nanomaterials and nanodevices used by the community. Furthermore, the information is not only gathered from specific nanoscience and nanotechnology journals, such as ours or Nano Letters, but also from journals such as Science, Angewandte Chemie International Edition and Advanced Materials, to list only a few.

Nano will grow to include more comprehensive information in the future. The plan for the rest of 2016 is to include information from a larger number of journals and to keep updating the database by including information from new publications. In the meantime, the product is ready to be used and we invite you to explore its functionalities, which can be done via institutional trials at http://nano.nature.com/. 